KOMPASS

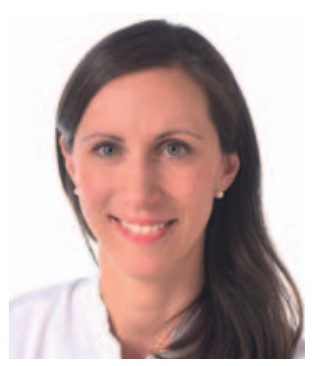

Sabine Steinke

Kompetenzzentrum Chronischer Pruritus,

Klinik für Hautkrankheiten,

Universitätsklinikum Münster,

Münster, Deutschland

Chronischer Pruritus ist ein häufiges Symptom, mit welchem Dermatologen, aber auch Internisten, Allgemeinmediziner, Neurologen und Gynäkologen häufig konfrontiert werden. Aktuellen Studien zufolge liegt in Deutschland eine Prävalenz von bis zu 17\% in der Allgemeinbevölkerung vor [1] Im deutschsprachigen Raum hat es sich daher vornehmlich die Arbeitsgemeinschaft Pruritusforschung (AGP) der Deutschen Dermatologischen Gesellschaft e.V. (DDG) zum Ziel gesetzt, das Symptom zu erforschen und die Diagnostik und Therapie voranzutreiben.

Chronischer Pruritus schränkt nachweislich die Lebensqualität ebenso stark ein wie chronischer Schmerz. Der Wunsch der Patienten, die Ursache für das Symptom zu finden sowie eine adäquate Therapie zu erhalten, ist groß und scheint sogar höher als bei anderen häufigen Hauterkrankungen wie der Neurodermitis oder der Psoriasis zu sein $[2,3]$. Der Pruritus ist häufig therapierefraktär und damit belastender als die Hauterkrankung selbst, wird aber in der Versorgung noch immer nicht ausreichend berücksichtigt oder gar nicht erfragt. Umso wichtiger ist es, die Bedeutung von Pruritus nicht nur für den Patienten, sondern auch als klinisches Merkmal, beispielsweise als (prämonitorisches) Symptom maligner Erkrankungen, zu kennen.

Dumont et al. beschreiben beispielhaft den Fall einer jungen Patientin, bei welcher ein

\title{
Chronischer Pruritus - eine interdisziplinäre Herausforderung
}

generalisierter Pruritus erst auf nichtentzündlicher Haut (International Forum for the Study of Itch(IFSI)-Klassifikation der Gruppe 2 [4]) und im Verlauf mit chronischen Kratzläsionen vom Typ einer chronischen nodulären Prurigo (IFSI-Klassifikation der Gruppe 3) Jahre vor der Diagnose eines Hodgkin-Lymphoms auftrat [5]. In unserem Kompetenzzentrum Chronischer Pruritus (KCP) in Münster sehen wir derartige Fälle gehäuft, insbesondere wenn Patienten mit kurzen Anamnesen (Dauer des Pruritus unter einem Jahr) zur Konsultation kommen. Ein standardisiertes Durchuntersuchungsprogramm umfasst in solchen Fällen stets auch ein orientierendes Abklären eines möglicherweise paraneoplastischen Pruritus, beispielsweise mittels Blutanalysen und radiologischen Methoden bzw. bei Anamnese einer B-Symptomatik direkt mittels Computertomografie. Oft geben diese Patienten aquagenen Pruritus (Pruritus nach Wasserkontakt) an. Eine gut organisierte interdisziplinäre Zusammenarbeit ist ein wichtiger Erfolgsfaktor in der raschen und korrekten Diagnosestellung.

Rowe und Yosipovitch erläutern in ihrer Übersichtsarbeit die neuesten Ansätze zur Behandlung des paraneoplastischen Pruritus [6]. Da dieser vermutlich nichthistaminvermittelt ist, sind Antihistaminika, welche bei Pruritus immer noch oft als erste Therapiestufe versucht werden, wenig hilfreich. Wirksamere Therapien setzen an zentralen Regulationsmechanismen wie der Modulation zentraler Opioidrezeptoren oder der Verringerung neuronaler Erregbarkeit an. Auch die Verringerung der Freisetzung bestimmter Neuropeptide wie z.B. Substanz P spielt eine therapeutische Rolle. Lesen Sie zu den Therapieempfehlungen gerne auch in der aktuellen S2k-Leitlinie nach [7].

Wir freuen uns sehr, dass sich die aktuelle Ausgabe des Karger Kompass Dermatologie diesem hochspannenden und interdisziplinär herausfordernden Thema der Paraneoplasien widmet und wünschen Ihnen viel Freude bei der Lektüre der interessanten Beiträge.

Mit herzlichen Grüßen aus Münster,
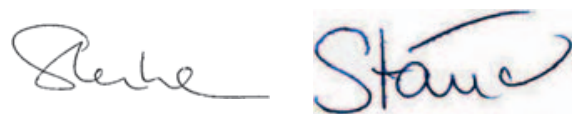

Ihre Sabine Steinke und Sonja Ständer

\section{Literatur}

1 Ständer S, Schäfer I, Phan NQ, et al.: Prevalence of chronic pruritus in Germany: results of a cross-sectional study in a sample working population of 11.730. Dermatology 2010;221: 229-235.

2 Steinke S, Bruland P, Blome C, et al.: Chronic pruritus: evaluation of patient needs and treatment goals with a special regard to differences according to pruritus classification and sex. $\mathrm{Br}$ J Dermatol 2017;176:363-370.

3 Steinke S, Blome C, Augustin M: Therapieziele und Therapienutzen bei dermatologischen $\mathrm{Pa}$ tienten. Akt Dermatol 2015;41:340-345.

4 Ständer S, Weisshaar E, Mettang T, et al.: Clinical classification of itch: a position paper of the International Forum for the Study of Itch. Acta Derm Venereol 2007;87:291-294.

5 Dumont S, Péchère M, Toutous Trellu L: Chronische Prurigo: eine ungewöhnliche Manifestation des Hodgkin-Lymphoms. Karger Kompass Dermatol 2018;4:227-229.

6 Rowe B, Yosipovitch G: Management von paraneoplastischem Pruritus. Karger Kompass Dermatol 2018;4:195-199.

7 Ständer S, Zeidler C, Augustin M, et al.: S2k Guidelines for the diagnosis and treatment of chronic pruritus - update - short version. J Dtsch Dermatol Ges. 2017;15:860-872.

\section{KARGER}

() 2018 S. Karger GmbH, Freiburg

Fax +4976145207 14

information@karger.com

www.karger.com
Accessible online at: www.karger.com/kkd
Dr. Sabine Steinke

Kompetenzzentrum Chronischer Pruritus, Klinik für Hautkrankheiten, Universitätsklinikum Münster

Von-Esmarch-Straße 58, 48149 Münster, Deutschland

sabine.steinke@ukmuenster.de 